PROCEEDINGS OF THE

AMERICAN MATHEMATICAL SOCIETY

Volume 140, Number 4, April 2012, Pages 1397-1402

S 0002-9939(2011)11033-5

Article electronically published on August 10, 2011

\title{
GENERALIZATION OF A THEOREM OF CLUNIE AND HAYMAN
}

\author{
MATTHEW BARRETT AND ALEXANDRE EREMENKO
}

(Communicated by Mario Bonk)

\begin{abstract}
Clunie and Hayman proved that if the spherical derivative $\left\|f^{\prime}\right\|$ of an entire function satisfies $\left\|f^{\prime}\right\|(z)=O\left(|z|^{\sigma}\right)$, then $T(r, f)=O\left(r^{\sigma+1}\right)$. We generalize this to holomorphic curves in projective space of dimension $n$ omitting $n$ hyperplanes in general position.
\end{abstract}

\section{INTRODUCTION}

We consider holomorphic curves $f: \mathbf{C} \rightarrow \mathbf{P}^{n}$; for the general background on the subject we refer to [7. The Fubini-Study derivative $\left\|f^{\prime}\right\|$ measures the length distortion from the Euclidean metric in $\mathbf{C}$ to the Fubini-Study metric in $\mathbf{P}^{n}$. The explicit expression is

$$
\left\|f^{\prime}\right\|^{2}=\|f\|^{-4} \sum_{i<j}\left|f_{i}^{\prime} f_{j}-f_{i} f_{j}^{\prime}\right|^{2},
$$

where $\left(f_{0}, \ldots, f_{n}\right)$ is a homogeneous representation of $f$ (that is, the $f_{j}$ are entire functions which never simultaneously vanish), and

$$
\|f\|^{2}=\sum_{j=0}^{n}\left|f_{j}\right|^{2} .
$$

See $[3$ for a general discussion of the Fubini-Study derivative.

We recall that the Nevanlinna-Cartan characteristic is defined by

$$
T(r, f)=\int_{0}^{r} \frac{d t}{t}\left(\frac{1}{\pi} \int_{|z| \leq t}\left\|f^{\prime}\right\|^{2}(z) d m(z)\right),
$$

where $d m$ is the area element in $\mathbf{C}$. So the condition

$$
\limsup _{z \rightarrow \infty}|z|^{-\sigma}\left\|f^{\prime}(z)\right\| \leq K<\infty
$$

implies

$$
\limsup _{r \rightarrow \infty} \frac{T(r, f)}{r^{2 \sigma+2}}<\infty
$$

Received by the editors November 17, 2010 and, in revised form, January 6, 2011.

2010 Mathematics Subject Classification. Primary 32Q99, 30D15.

The first and second authors are supported by NSF grant DMS-0555279.

The second author is also supported by the Humboldt Foundation.

(C)2011 American Mathematical Society Reverts to public domain 28 years from publication 
Clunie and Hayman [4] found that for curves $\mathbf{C} \rightarrow \mathbf{P}^{1}$ omitting one point in $\mathbf{P}^{1}$, a stronger conclusion follows from (1), namely

$$
\limsup _{r \rightarrow \infty} \frac{T(r, f)}{r^{\sigma+1}} \leq K C(\sigma) .
$$

In the most important case of $\sigma=0$, a different proof of this fact for $n=1$ is due to Pommerenke [8]. Pommerenke's method gives the exact constant $C(0)$. In this paper we prove that this phenomenon persists in all dimensions.

Theorem. For holomorphic curves $f: \mathbf{C} \rightarrow \mathbf{P}^{n}$ omitting $n$ hyperplanes in general position, condition (1) implies (3) with an explicit constant $C(n, \sigma)$.

In [6], the case $\sigma=0$ was considered. There it was proved that holomorphic curves in $\mathbf{P}^{n}$ with bounded spherical derivative and omitting $n$ hyperplanes in general position must satisfy $T(r, f)=O(r)$. With a stronger assumption that $f$ omits $n+1$ hyperplanes this was earlier established by Berteloot and Duval [2] and by Tsukamoto [9]. The proof in [6] has two drawbacks: it does not extend to arbitrary $\sigma \geq 0$, and it is non-constructive; unlike Clunie-Hayman and Pommerenke's proofs mentioned above, it does not give an explicit constant in (3).

It is shown in 6] that the condition that $n$ hyperplanes are omitted is exact: there are curves in any dimension $n$ satisfying (11), $T(r, f) \sim c r^{2 \sigma+2}$ and omitting $n-1$ hyperplanes.

\section{Preliminaries}

Without loss of generality we assume that the omitted hyperplanes are given in the homogeneous coordinates by the equations $\left\{w_{j}=0\right\}, 1 \leq j \leq n$. We fix a homogeneous representation $\left(f_{0}, \ldots, f_{n}\right)$ of our curve, where $f_{j}$ are entire functions and $f_{n}=1$. Then

$$
u=\log \sqrt{\left|f_{0}\right|^{2}+\ldots+\left|f_{n}\right|^{2}}
$$

is a positive subharmonic function, and Jensen's formula gives

$$
T(r, f)=\frac{1}{2 \pi} \int_{-\pi}^{\pi} u\left(r e^{i \theta}\right) d \theta-u(0)=\int_{0}^{r} \frac{n(t)}{t} d t
$$

where $n(t)=\mu(\{z:|z| \leq t\})$ and $\mu=\mu_{u}$ is the Riesz measure of $u$, that is, the measure with the density

$$
\frac{1}{2 \pi} \Delta u=\frac{1}{\pi}\left\|f^{\prime}\right\|^{2}
$$

This measure $\mu$ is also called Cartan's measure of $f$. Positivity of $u$ and (2) imply that all $f_{j}$ are of order at most $2 \sigma+2$, normal type. As $f_{j}(z) \neq 0,1 \leq j \leq n$, we conclude that

$$
f_{j}=e^{P_{j}}, \quad 1 \leq j \leq n,
$$

where

$$
P_{j} \text { are polynomials of degree at most } 2 \sigma+2 .
$$

We need two lemmas from potential theory.

Lemma 1 ([6]). Let $v$ be a non-negative harmonic function in the closure of the disc $B(a, R)$, and assume that $v\left(z_{1}\right)=0$ for some point $z_{1} \in \partial B(a, R)$. Then

$$
v(a) \leq 2 R\left|\nabla v\left(z_{1}\right)\right| \text {. }
$$


We include a proof, suggested by the referee, which is simpler than that given in 6]. Without loss of generality, assume that $a=0, R=1, z_{1}=1$. Then Harnack's inequality gives

$$
\frac{v(0)}{1+r} \leq \frac{v(r)}{1-r}=\frac{v(r)-v(1)}{1-r}
$$

Passing to the limit as $r \rightarrow 1$, we obtain the result.

Lemma 2. Let $v$ be a non-negative superharmonic function in the closure of the disc $B(a, R)$ and suppose that $v\left(z_{1}\right)=0$ for some $z_{1} \in \partial B(a, R)$. Then

$$
\left|\mu_{v}(B(a, R / 2))\right| \leq 3 R\left|\frac{\partial v}{\partial n}\left(z_{1}\right)\right| .
$$

By $|\partial v / \partial n|$ we mean here $\liminf \left|v\left(r z_{1}\right)\right| /(R(1-r))$ as $r \rightarrow 1-$.

Proof. The function $v(a+R z)$ satisfies the conditions of the lemma with $R=1$. So it is enough to prove the lemma with $a=0$ and $R=1$. Let

$$
w(z)=\int_{|\zeta| \leq 1 / 2} G(z, \zeta) d \mu_{v}(\zeta)
$$

be the Green potential of the restriction of $\mu_{v}$ onto the disc $|\zeta| \leq 1 / 2$, that is,

$$
G(z, \zeta)=\log \left|\frac{1-\bar{\zeta} z}{z-\zeta}\right| .
$$

Then $w \leq v$ and $w\left(z_{1}\right)=v\left(z_{1}\right)=0$, which implies that

$$
\left|\frac{\partial v}{\partial n}\left(z_{1}\right)\right| \geq\left|\frac{\partial w}{\partial|z|}\left(z_{1}\right)\right| .
$$

Minimizing $|\partial G / \partial| z||$ over $|z|=1$ and $|\zeta|=1 / 2$, we obtain $1 / 3$, which proves the lemma.

\section{ProOF OF THE THEOREM}

We may assume without loss of generality that $f_{0}$ has infinitely many zeros. Indeed, we can compose $f$ with an automorphism of $\mathbf{P}^{n}$; for example replace $f_{0}$ by $f_{0}+c f_{1}, c \in \mathbf{C}$, and leave all other $f_{j}$ unchanged. This transformation changes neither the $n$ omitted hyperplanes nor the rate of growth of $T(r, f)$ and multiplies the spherical derivative by a bounded factor.

Let $u_{j}=\log \left|f_{j}\right|$ and

$$
u^{*}=\max _{1 \leq j \leq n} u_{j} .
$$

Here and in what follows max denotes the pointwise maximum of subharmonic functions.

Proposition 1. Suppose that at some point $z_{1}$ we have

$$
u_{m}\left(z_{1}\right)=u_{k}\left(z_{1}\right) \geq u_{j}\left(z_{1}\right)
$$

for some $m \neq k$ and all $j$ where $m, k, j \in\{0, \ldots, n\}$. Then

$$
\left\|f^{\prime}\left(z_{1}\right)\right\| \geq(n+1)^{-1}\left|\nabla u_{m}\left(z_{1}\right)-\nabla u_{k}\left(z_{1}\right)\right| .
$$


Proof.

$$
\left\|f^{\prime}\left(z_{1}\right)\right\| \geq \frac{\left|f_{m}^{\prime}\left(z_{1}\right) f_{k}\left(z_{1}\right)-f_{m}\left(z_{1}\right) f_{k}^{\prime}\left(z_{1}\right)\right|}{\left|f_{0}\left(z_{1}\right)\right|^{2}+\ldots+\left|f_{n}\left(z_{1}\right)\right|^{2}} \geq(n+1)^{-1}\left|\frac{f_{m}^{\prime}\left(z_{1}\right)}{f_{m}\left(z_{1}\right)}-\frac{f_{k}^{\prime}\left(z_{1}\right)}{f_{k}\left(z_{1}\right)}\right|,
$$

and the conclusion of the proposition follows since $|\nabla \log | f||=\left|f^{\prime} / f\right|$.

Proposition 2. For every $\epsilon>0$, we have

$$
u(z) \leq u^{*}(z)+K(2+\epsilon)^{\sigma+1}(n+1)|z|^{\sigma+1}
$$

for all $|z|>r_{0}(\epsilon)$.

Proof. If $u_{0}(z) \leq u^{*}(z)$ for all sufficiently large $|z|$, then there is nothing to prove. Suppose that $u_{0}(a)>u^{*}(a)$, and consider the largest disc $B(a, R)$ centered at $a$ where the inequality $u_{0}(z)>u^{*}(z)$ persists. If $z_{0}$ is the zero of the smallest modulus of $f_{0}$, then $R \leq|a|+\left|z_{0}\right|<(1+\epsilon)|a|$ when $|a|$ is large enough.

There is a point $z_{1} \in \partial B(a, R)$ such that $u_{0}\left(z_{1}\right)=u^{*}\left(z_{1}\right)$. This means that there is some $k \in\{1, \ldots, n\}$ such that $u_{0}\left(z_{1}\right)=u_{k}\left(z_{1}\right) \geq u_{m}\left(z_{1}\right)$ for all $m \in\{1, \ldots, n\}$. Applying Proposition 1 we obtain

$$
\left|\nabla u_{k}\left(z_{1}\right)-\nabla u_{0}\left(z_{1}\right)\right| \leq(n+1)\left\|f^{\prime}\left(z_{1}\right)\right\| .
$$

Now $u_{0}(z)>u^{*}(z) \geq u_{k}(z)$ for $z \in B(a, R)$, so we can apply Lemma 1 to $v=u_{0}-u_{k}$ in the disc $B(a, R)$. This gives

$$
u_{0}(a)-u_{k}(a) \leq 2 R\left|\nabla u_{k}\left(z_{1}\right)-\nabla u_{0}\left(z_{1}\right)\right| \leq 2 R(n+1)\left\|f^{\prime}\left(z_{1}\right)\right\| .
$$

Now $R<(1+\epsilon)|a|$ and $\left|z_{1}\right| \leq(2+\epsilon)|a|$, so

$$
u_{0}(a) \leq u^{*}(a)+K(2+\epsilon)^{\sigma+1}(n+1)|a|^{\sigma+1},
$$

and the result follows because $u=\max \left\{u_{0}, u^{*}\right\}+O(1)$.

Next we study the Riesz measure of the subharmonic function

$$
u^{*}=\max \left\{u_{1}, \ldots, u_{n}\right\} .
$$

We begin with the maximum of two harmonic functions. Let $u_{1}$ and $u_{2}$ be two harmonic functions in $\mathbf{C}$ of the form $u_{j}=\operatorname{Re} P_{j}$ where $P_{j} \neq 0$ are polynomials. Suppose that $u_{1} \neq u_{2}$. Then the set $E=\left\{z \in \mathbf{C}: u_{1}(z)=u_{2}(z)\right\}$ is a proper realalgebraic subset of $\overline{\mathbf{C}}$ without isolated points. Apart from a finite set of ramification points, $E$ consists of smooth curves. For every smooth point $z \in E$, we denote by $J(z)$ the jump of the normal (to $E$ ) derivative of the function $w=\max \left\{u_{1}, u_{2}\right\}$ at the point $z$. This jump is always positive and the Riesz measure $\mu_{w}$ is given by the formula

$$
d \mu_{w}=\frac{J(z)}{2 \pi}|d z|
$$

which means that $\mu_{w}$ is supported by $E$ and has a density $J(z) / 2 \pi$ with respect to the length element $|d z|$ on $E$.

Now let $E_{i, j}=\left\{z: u_{i}(z)=u_{j}(z) \geq u_{k}(z), 1 \leq k \leq n\right\}$, and let $E=\bigcup E_{i, j}$ where the union is taken over all pairs $1 \leq i, j \leq n$ for which $u_{i} \neq u_{j}$. Then $E$ is a proper real semi-algebraic subset of $\overline{\mathbf{C}}$ and $\infty$ is not an isolated point of $E$. For the elementary properties of semi-algebraic sets that we use here, see, for example, 
[1, [5]. There exists $r_{0}>0$ such that $\Gamma=E \cap\left\{r_{0}<|z|<\infty\right\}$ is a union of finitely many disjoint smooth simple curves,

$$
\Gamma=\bigcup_{k=1}^{m} \Gamma_{k} .
$$

This union coincides with the support of $\mu_{u^{*}}$ in $\left\{z: r_{0}<|z|<\infty\right\}$.

Consider a point $z_{0} \in \Gamma$. Then $z_{0} \in \Gamma_{k}$ for some $k$. As $\Gamma_{k}$ is a smooth curve, there is a neighborhood $D$ of $z_{0}$ which does not contain other curves $\Gamma_{j}, j \neq k$, and which is divided by $\Gamma_{k}$ into two parts, $D_{1}$ and $D_{2}$. Then there exist $i$ and $j$ such that $u^{*}(z)=u_{i}(z), z \in D_{1}$ and $u^{*}(z)=u_{j}(z), z \in D_{2}$, and $u^{*}(z)=$ $\max \left\{u_{i}(z), u_{j}(z)\right\}, z \in D$. So the restriction of the Riesz measure $\mu_{u^{*}}$ on $D$ is supported by $\Gamma_{k} \cap D$ and has density $J(z) /(2 \pi)$ where

$$
|J(z)|=\left|\partial u_{i} / \partial n-\partial u_{j} / \partial n\right|(z)=\left|\nabla\left(u_{i}-u_{j}\right)\right|(z)
$$

and $\partial / \partial n$ is the derivation in the direction of a normal to $\Gamma_{k}$. Taking into account that $u_{j}=\operatorname{Re} P_{j}$ where $P_{j}$ are polynomials, we conclude that there exist positive numbers $c_{k}$ and $b_{k}$ such that

$$
J(z) /(2 \pi)=\left(c_{k}+o(1)\right)|z|^{b_{k}}, \quad z \rightarrow \infty, \quad z \in \Gamma_{k} .
$$

Let $b=\max _{k} b_{k}$, and among those curves $\Gamma_{k}$ for which $b_{k}=b$ choose one with maximal $c_{k}$ (which we denote by $c_{0}$ ). We denote this chosen curve by $\Gamma_{0}$ and fix it for the rest of the proof.

Proposition 3. We have

$$
b \leq \sigma \quad \text { and } \quad c_{0} \leq 3 \cdot 4^{\sigma} K(n+1) .
$$

Proof. We consider two cases.

Case 1. There is a sequence $z_{n} \rightarrow \infty, z_{n} \in \Gamma_{0}$, such that $u_{0}\left(z_{n}\right) \leq u^{*}\left(z_{n}\right)$. Then (11) and Proposition 1 imply that

$$
J\left(z_{n}\right) \leq(n+1) K\left|z_{n}\right|^{\sigma},
$$

and comparison with (8) shows that $b \leq \sigma$ and $c_{0} \leq K(n+1) /(2 \pi)$.

Case 2. $u_{0}(z)>u^{*}(z)$ for all sufficiently large $z \in \Gamma_{0}$. Let $a$ be a point on $\Gamma_{0}$, $|a|>3 r_{0}$, and $u_{0}(a)>u^{*}(a)$. Let $B(a, R)$ be the largest open disc centered at $a$ in which the inequality $u_{0}(z)>u^{*}(z)$ holds. Then

$$
R \leq|a|+O(1), \quad a \rightarrow \infty,
$$

because we assume that $f_{0}$ has zeros, so $u_{0}\left(z_{0}\right)=-\infty$ for some $z_{0}$.

In $B(a, R)$ we consider the positive superharmonic function $v=u_{0}-u^{*}$. Let us check that it satisfies the conditions of Lemma 2. The existence of a point $z_{1} \in \partial B(a, R)$ with $v\left(z_{1}\right)=0$ follows from the definition of $B(a, R)$. The Riesz measure of $\mu_{v}$ is estimated using (7), (8):

$$
\left|\mu_{v}(B(a, R / 2))\right| \geq\left|\mu_{v}\left(\Gamma_{0} \cap B(a, R / 2)\right)\right| \geq c_{0} R(|a|-R / 2)^{b} .
$$

Now Lemma 2 applied to $v$ in $B(a, R)$ implies that

$$
\left|\nabla v\left(z_{1}\right)\right| \geq\left(c_{0} / 3\right)(|a|-R / 2)^{b} .
$$

On the other hand (11) and Proposition 1 imply that

$$
\left|\nabla v\left(z_{1}\right)\right| \leq K(n+1)(|a|+R)^{\sigma} .
$$


Combining these two inequalities and taking (9) into account, we obtain $b \leq \sigma$ and $c_{0} \leq 3 \cdot 4^{\sigma} K(n+1)$, as required.

We denote

$$
T^{*}(r)=\frac{1}{2 \pi} \int_{-\pi}^{\pi} u^{*}\left(r e^{i \theta}\right) d \theta-u^{*}(0) .
$$

This is the characteristic of the "reduced curve" $\left(f_{1}, \ldots, f_{n}\right)$.

\section{Proposition 4.}

$$
T^{*}(r) \leq 6 \cdot 4^{\sigma} K \frac{n(n+1)^{2}}{\sigma+1} r^{\sigma+1} .
$$

Proof. By Jensen's formula,

$$
T^{*}(r)=\int_{0}^{r} \nu(t) \frac{d t}{t}
$$

where $\nu(t)=\mu_{u^{*}}(\{z:|z| \leq t\})$. The number of curves $\Gamma_{k}$ supporting the Riesz measure of $u^{*}$ is easily seen to be at most $2 n(n-1)(\sigma+1)$. The density of the Riesz measure $\mu_{u^{*}}$ on each curve $\Gamma_{k}$ is given by (8), where $c_{k} \leq c_{0}$ and $b_{k} \leq b$ and the parameters $c_{0}$ and $b$ are estimated in Proposition 3. Combining all these data, we obtain the result.

It remains to combine Propositions 2 and 4 to obtain the final result.

\section{ACKNOWLEDGMENT}

The authors thank the referee for many valuable remarks and suggestions.

\section{REFERENCES}

[1] R. Benedetti and J. Risler, Real algebraic and semi-algebraic sets, Hermann, Paris, 1990. MR:1070358 (91j:14045)

[2] F. Berteloot and J. Duval, Sur l'hyperbolicité de certains complémentaires, Enseign. Math. (2) 47 (2001), no. 3-4, 253-267. MR1876928 (2002m:32042)

[3] W. Cherry and A. Eremenko, Landau's theorem for holomorphic curves in projective space and the Kobayashi metric on hyperplane complement, Pure and Appl. Math. Quarterly 7 (2011), no. 1, 199-221.

[4] J. Clunie and W. Hayman, The spherical derivative of integral and meromorphic functions, Comment. Math. Helv. 40 (1966) 117-148. MR0192055 (33:282)

[5] M. Coste, An introduction to semialgebraic geometry, Inst. editoriali e poligrafici internazionali, Pisa, 2000.

[6] A. Eremenko, Brody curves omitting hyperplanes, Ann. Acad. Sci. Fenn. 35 (2010) 565-570. MR2731707

[7] S. Lang, Introduction to complex hyperbolic spaces, Springer-Verlag, New York, 1987. MR886677 (88f:32065)

[8] Ch. Pommerenke, Estimates for normal meromorphic functions, Ann. Acad. Sci. Fenn. Ser. A I 476 (1970). MR0285710(44:2928)

[9] M. Tsukamoto, On holomorphic curves in algebraic torus, J. Math. Kyoto Univ. 47 (2007), no. 4, 881-892. MR.2413072(2009a:32023)

Department of Mathematics, Purdue University, West Lafayette, Indiana 47907

Department of Mathematics, Purdue University, West Lafayette, Indiana 47907

E-mail address: eremenko@math.purdue.edu 